\title{
More than ramps: accessible health care for people with disabilities
}

$\mathrm{O}$ ne in 8 Canadians - a number in excess of 3.5 million people - lives with a disability. ${ }^{1}$ Advances in the provision of acute health care and an aging population mean that the number of people in Canada with disabilities will continue to grow. Yet little attention is directed toward the needs of this large patient population.

Recent discussions on health care in Canada have focused on 2 principal areas: the use of private services and the potential emergence of a 2-tier health system, and wait times for services. However, to consider the accessibility of health care for people with disabilities is to see that Canada already has a 2-tier health system. As important as timely access to care may be, of prime concern to this patient population is their access to medically necessary care.

In spite of their potential complexity, many of the basic health care needs of people with disabilities are the same as those of the general population. Yet people with disabilities do not receive the same level of primary and preventive care as others $\mathrm{do}^{2}$ Routine interventions such as a Pap smear or prostate exam are not consistently provided to them. Even more disturbing, people with disabilities are 4 times as likely as able-bodied people to report an inability to obtain required medical care when it is needed. ${ }^{2}$

Since disabilities related to patient mobility are among the most common in Canada, physical access is an obvious concern. ${ }^{1}$ Even when a building or office permits wheelchair access, one has only to observe the standard examination table used by physicians to understand that most such offices are not truly accessible. This may help explain why patients with physical disabilities often do not receive proper examinations. New legislation such as the 2005 Accessibility for Ontarians with Disabilities Act considers accessibility in hospitals but does not address physicians' offices. Nevertheless, lack of legislation does not prevent physicians from following available guidelines to make their offices accessible to their patients.

The removal of environmental barriers alone is insufficient to ensure accessible health care. Medical training focuses much more on curing and preventing disease than on the management of patients with chronic diseases. Many will rejoice at a life saved, of course, but the provision of long-term care is rarely celebrated. Students and residents finish their training with only limited time spent on the provision of $\curvearrowleft$ chronic care, and many remain unsure about how to interact with patients with disabilities.

With current pressures to discharge patients from acute care facilities, timely access to rehabilitation services should not be at issue. Yet access to rehabilitation programs is frequently lacking, despite evidence that such delays significantly reduce the functional recovery of patients. ${ }^{3}$ Wait-lists for selected procedures have received attention that reflects political agendas as much as solid clinical evidence. No one can argue against the benefits of timely availability of joint replacements to those with degenerative joint disease, or of tissue plasminogen activator (tPA) to acute stroke patients. But because physician resources are limited, increased use of services for selected procedures can change the service mix provided and unintentionally cause even longer wait-lists for equally impaired patients with chronic disabilities. Although wait times for non-select procedures have not been tracked, in our experience waiting periods for non-select orthopedic assessments have lengthened since wait-list times for joint replacements have become a priority. Similarly, resources for stroke rehabilitation have not matched the resources made available for tPA administration.

Patients with disabilities often have complex medical conditions requiring that multiple issues be addressed when they visit a physician. They may also require additional time and assistance to move about and communicate. Some provinces have a fee code for chronic-care services that partially compensates physicians for the extra time required. However, until adequate numbers of physicians are available and fee-forservice physicians feel adequately compensated, patients with disabilities are likely to continue to have difficulty obtaining comprehensive care.

People with disabilities clearly need improved access to quality health care. In addition to interventions at the system level, we can individually improve the care we provide. Together, we can make a difference to the health of this very important and growing group of patients.

\section{Meridith B. Marks}

Physical Medicine and Rehabilitation

University of Ottawa, Ottawa, Ont.

Robert Teasell

Physical Medicine and Rehabilitation

University of Western Ontario, London, Ont.

This article has been peer reviewed.

\section{REFERENCES}

I. Office for Disability Issues, Social Development Canada. Advancing the inclusion of persons with disabilities 2004: a Government of Canada report. Ottawa: Government of Canada; 2004. Available: www.sdc.gc.ca/en/hip/odi/documents /advancingInclusiono4/aipdo4.pdf (accessed 2006 Jul ro).

2. Canadian Council on Social Development. CCSD's disability information sheet: No. 9, 2003. The health and well-being of persons with disabilities. Available: www.ccsd.ca/drip/research/dis9/index.htm (accessed $2006 \mathrm{Jul}$ Io).

3. Salter K, Jutai J, Hartley M, et al. Impact of early vs delayed admission to rehabilitation on functional outcomes in persons with stroke. JRehabil Med 2006;38:113-7. 